\title{
Synthetic Approaches to Guanacastepenes. Enantiospecific Syntheses of BC and AB Ring Systems of Guanacastepenes and Rameswaralide
}

\author{
A. Srikrishna* and Dattatraya H. Dethe
}

\section{Supporting Information}

IR spectra were recorded on Jasco FTIR 410 spectrophotometer. ${ }^{1} \mathrm{H}(300 \mathrm{MHz})$ and ${ }^{13} \mathrm{C}$ (75 MHz) NMR spectra were recorded on Jeol JNM $\lambda$-300 spectrometer using 1:1 mixture of $\mathrm{CDCl}_{3}$ and $\mathrm{CCl}_{4}$ as the solvent.. The chemical shifts $(\delta \mathrm{ppm})$ and coupling constants $(\mathrm{Hz})$ are reported in the standard fashion with reference to either internal tetramethylsilane (for ${ }^{1} \mathrm{H}$ ) or the central line $\left(77.0 \mathrm{ppm}\right.$ ) of $\mathrm{CDCl}_{3}$ (for ${ }^{13} \mathrm{C}$ ). In the ${ }^{13} \mathrm{C}$ NMR spectra, the nature of the carbons $(\mathrm{C}$, $\mathrm{CH}, \mathrm{CH}_{2}$ or $\mathrm{CH}_{3}$ ) was determined by recording the DEPT-135 spectrum, and is given in parentheses. Low-resolution mass spectra were recorded using Shimadzu GCMS-QP5050A instrument using direct inlet mode. Relative intensities are given in parentheses. High-resolution

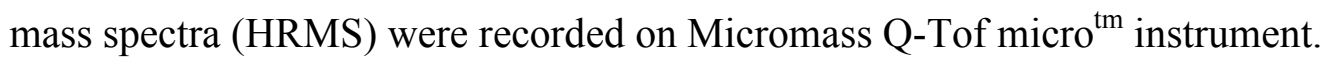


(-)-(5S,6R)-6-Allyl-2,6-dimethyl-5-isopropenylcyclohex-2-enone (5):

$[\alpha]_{D}^{23}:-40.0\left(c 2.5, \mathrm{CHCl}_{3}\right)$.

$\mathrm{UV} / \mathrm{Vis}\left(\mathrm{CH}_{3} \mathrm{CN}\right): \lambda_{\max }=236(\epsilon=9008)$.

IR (neat): $v_{\max } / \mathrm{cm}^{-1} 3076,2974,2923,1670,1639,1438,1376,1220,1194,1078,1028,993$, 911, 900 .

${ }^{1} \mathrm{H}$ NMR $\left(300 \mathrm{MHz}, \mathrm{CDCl}_{3}+\mathrm{CCl}_{4}\right): \delta 6.53(1 \mathrm{H}$, br s), 5.75-5.57 $(1 \mathrm{H}, \mathrm{m})$, 5.06-4.92 $(2 \mathrm{H}, \mathrm{m})$, 4.87 (1 H, s), $4.73(1 \mathrm{H}, \mathrm{s}), 2.57(1 \mathrm{H}, \mathrm{t}, \mathrm{J}=6.0 \mathrm{~Hz}), 2.50-2.30(3 \mathrm{H}, \mathrm{m}), 2.15(1 \mathrm{H}, \mathrm{dd}, \mathrm{J}=13.5$ and $7.5 \mathrm{~Hz}), 1.76(3 \mathrm{H}, \mathrm{s})$ and $1.71(3 \mathrm{H} \mathrm{s})$ [2 x olefinic $\left.\mathrm{CH}_{3}\right], 1.09\left(3 \mathrm{H}, \mathrm{s}\right.$, tert- $\left.\mathrm{CH}_{3}\right)$.

${ }^{13} \mathrm{C}$ NMR $\left(75 \mathrm{MHz}, \mathrm{CDCl}_{3}+\mathrm{CCl}_{4}\right): \delta 202.8(\mathrm{C}, \mathrm{C}=\mathrm{O}), 145.4(\mathrm{C}), 140.7(\mathrm{CH}), 134.4(\mathrm{CH}), 134.1$ (C), $117.8\left(\mathrm{CH}_{2}\right), 114.3\left(\mathrm{CH}_{2}\right), 51.0(\mathrm{CH}, \mathrm{C}-5), 47.4(\mathrm{C}, \mathrm{C}-6), 37.2\left(\mathrm{CH}_{2}\right), 29.1\left(\mathrm{CH}_{2}\right), 23.6$ $\left(\mathrm{CH}_{3}\right), 21.6\left(\mathrm{CH}_{3}\right), 16.6\left(\mathrm{CH}_{3}\right)$.

HRMS: For $\mathrm{C}_{14} \mathrm{H}_{20} \mathrm{ONa}(\mathrm{M}+\mathrm{Na})$, Calcd.: 227.1412. Found: 227.1410.

\section{(-)-(5S,6S)-6-Allyl-2,6-dimethyl-5-isopropenylcyclohex-2-enone (6):}

$[\alpha]_{D}^{22}:-45.9\left(c 5.8, \mathrm{CHCl}_{3}\right)$.

IR (neat): $v_{\max } / \mathrm{cm}^{-1} 3076,2977,1667,1644,1450,1377,997,913,900$.

${ }^{1} \mathrm{H}$ NMR (300 MHz, $\left.\mathrm{CDCl}_{3}+\mathrm{CCl}_{4}\right): \delta 6.54(1 \mathrm{H}, \mathrm{s}), 5.69(1 \mathrm{H}, \mathrm{t}$ of dd, J = 17.1, 9.9 and $7.2 \mathrm{~Hz})$, 5.07-4.98 (2 H, m), $4.75(1 \mathrm{H}, \mathrm{s}), 4.70(1 \mathrm{H}, \mathrm{s}), 2.72-2.58(2 \mathrm{H}, \mathrm{m}), 2.36(1 \mathrm{H}, \mathrm{dd}, \mathrm{J}=13.2$ and $7.5 \mathrm{~Hz}), 2.33-2.30(1 \mathrm{H}, \mathrm{m}), 2.21(1 \mathrm{H}, \mathrm{dd}, \mathrm{J}=13.2$ and $6.6 \mathrm{~Hz}), 1.76(3 \mathrm{H}, \mathrm{s})$ and $1.62(3 \mathrm{H}, \mathrm{s})[2$ x olefinic $\left.\mathrm{CH}_{3}\right], 1.01\left(3 \mathrm{H}\right.$, s, tert $\left.-\mathrm{CH}_{3}\right)$.

${ }^{13} \mathrm{C} \mathrm{NMR}\left(75 \mathrm{MHz}, \mathrm{CDCl}_{3}+\mathrm{CCl}_{4}\right): \delta 202.6(\mathrm{C}, \mathrm{C}=\mathrm{O}), 146.1(\mathrm{C}), 140.8(\mathrm{CH}), 134.3(\mathrm{C}), 134.2$ $(\mathrm{CH}), 118.1\left(\mathrm{CH}_{2}\right), 114.3\left(\mathrm{CH}_{2}\right), 49.6(\mathrm{CH}), 47.6(\mathrm{C}), 42.2\left(\mathrm{CH}_{2}\right), 28.6\left(\mathrm{CH}_{2}\right), 22.2\left(\mathrm{CH}_{3}\right), 19.2$ $\left(\mathrm{CH}_{3}\right), 16.5\left(\mathrm{CH}_{3}\right)$.

HRMS: For $\mathrm{C}_{14} \mathrm{H}_{20} \mathrm{ONa}(\mathrm{M}+\mathrm{Na})$, Calcd.: 227.1412. Found: 227.1431

(+)-(4R,5S)-4-Allyl-3-(but-3-enyl)-2,4-dimethyl-5-isopropenylcyclohex-2-enone (9): $[\alpha]_{D}^{23}: 76.3\left(c 1.9, \mathrm{CHCl}_{3}\right)$.

$\mathrm{UV} / \mathrm{V}$ is $\left(\mathrm{CH}_{3} \mathrm{CN}\right): \lambda_{\max }=250(\epsilon=10005)$.

IR (neat): $v_{\max } / \mathrm{cm}^{-1} 3076,2975,1667,1640,1609,1440,1377,1348,1310,1168,994,912$. ${ }^{1} \mathrm{H}$ NMR (300 MHz, $\left.\mathrm{CDCl}_{3}+\mathrm{CCl}_{4}\right): \delta$ 5.90-5.65 (2 H, m), 5.15-4.95 (4 H, m), $4.96(1 \mathrm{H}, \mathrm{s}), 4.80$ 
$(1 \mathrm{H}, \mathrm{s}), 2.70-2.10(9 \mathrm{H}, \mathrm{m}), 1.81(3 \mathrm{H}, \mathrm{s})$ and $1.76(3 \mathrm{H}, \mathrm{s})\left[2 \mathrm{x}\right.$ olefinic $\left.\mathrm{CH}_{3}\right], 1.22(3 \mathrm{H}, \mathrm{s}$, tert$\left.\mathrm{CH}_{3}\right)$.

${ }^{13} \mathrm{C}$ NMR $\left(75 \mathrm{MHz}, \mathrm{CDCl}_{3}+\mathrm{CCl}_{4}\right): \delta 197.5(\mathrm{C}, \mathrm{C}=\mathrm{O}), 161.8(\mathrm{C}, \mathrm{C}-3), 145.2\left(\mathrm{C}, \mathrm{C}=\mathrm{CH} \mathrm{H}_{2}\right), 137.4$ $(\mathrm{CH}), 134.7(\mathrm{CH}), 132.5(\mathrm{C}), 117.9\left(\mathrm{CH}_{2}\right), 115.4\left(\mathrm{CH}_{2}\right), 115.3\left(\mathrm{CH}_{2}\right), 51.3(\mathrm{CH}), 42.8(\mathrm{C}), 40.5$ $\left(\mathrm{CH}_{2}\right), 39.7\left(\mathrm{CH}_{2}\right), 32.6\left(\mathrm{CH}_{2}\right), 31.0\left(\mathrm{CH}_{2}\right), 25.1\left(\mathrm{CH}_{3}\right), 24.1\left(\mathrm{CH}_{3}\right), 11.7\left(\mathrm{CH}_{3}\right)$.

HRMS: For $\mathrm{C}_{18} \mathrm{H}_{26} \mathrm{ONa}(\mathrm{M}+\mathrm{Na})$, Calcd.: 281.1881. Found: 281.1892.

(+)-(4S,5S)-4-Allyl-3-(but-3-enyl)-2,4-dimethyl-5-isopropenylcyclohex-2-enone (10): $[\alpha]_{D}^{23}:+20.0\left(c 1.45, \mathrm{CHCl}_{3}\right)$.

$\mathrm{UV}\left(\mathrm{CH}_{3} \mathrm{CN}\right): \lambda_{\max }=250(\epsilon=8643)$.

IR (neat): $v_{\max } / \mathrm{cm}^{-1} 3076,2975,2921,1665,1640,1608,1440,1417,1377,1347,1309,1168$, 1080, 995, 912.

${ }^{1} \mathrm{H}$ NMR (300 MHz, $\left.\mathrm{CDCl}_{3}+\mathrm{CCl}_{4}\right):$ 5 5.90-5.60 (2 H, m), 5.15-4.95 (4 H, m), $4.81(1 \mathrm{H}, \mathrm{s}), 4.73$ $(1 \mathrm{H}, \mathrm{s}), 2.72(1 \mathrm{H}, \mathrm{t}, \mathrm{J}=5.7 \mathrm{~Hz}), 2.62(1 \mathrm{H}, \mathrm{ddd}, \mathrm{J}=17.4,5.7$ and $1.2 \mathrm{~Hz}), 2.45-2.10(7 \mathrm{H}, \mathrm{m})$, $1.81(3 \mathrm{H}, \mathrm{s})$ and $1.62(3 \mathrm{H}, \mathrm{s})\left[2 \mathrm{x}\right.$ olefinic $\left.\mathrm{CH}_{3}\right], 1.11\left(3 \mathrm{H}\right.$, s, tert $\left.-\mathrm{CH}_{3}\right)$.

${ }^{13} \mathrm{C} \mathrm{NMR}\left(75 \mathrm{MHz}, \mathrm{CDCl}_{3}+\mathrm{CCl}_{4}\right): \delta 197.0(\mathrm{C}, \mathrm{C}=\mathrm{O}), 160.8(\mathrm{C}, \mathrm{C}-3), 145.9\left(\mathrm{C}, \mathrm{C}=\mathrm{CH}_{2}\right), 137.4$ $(\mathrm{CH}), 134.1(\mathrm{CH}), 132.9(\mathrm{C}, \mathrm{C}-2), 118.5\left(\mathrm{CH}_{2}\right), 115.2\left(2 \mathrm{C}, \mathrm{CH}_{2}\right), 47.9(\mathrm{CH}, \mathrm{C}-5), 44.3\left(\mathrm{CH}_{2}\right)$, $42.2(\mathrm{C}), 38.9\left(\mathrm{CH}_{2}\right), 32.7\left(\mathrm{CH}_{2}\right), 30.4\left(\mathrm{CH}_{2}\right), 22.5\left(\mathrm{CH}_{3}\right), 21.4\left(\mathrm{CH}_{3}\right), 11.8\left(\mathrm{CH}_{3}\right)$. HRMS: For $\mathrm{C}_{18} \mathrm{H}_{26} \mathrm{ONa}(\mathrm{M}+\mathrm{Na})$, Calcd.: 281.1881. Found: 281.1891.

(+)-(1R,11S)-1,8-Dimethyl-11-isopropenylbiocyclo[5.4.0] undeca-3,7-diene-9-one (11): $[\alpha]_{D}^{23}:+2.95\left(c 1.7, \mathrm{CHCl}_{3}\right)$.

$\mathrm{UV} / \mathrm{Vis}\left(\mathrm{CH}_{3} \mathrm{CN}\right): \lambda_{\max }=250.6(\epsilon=8672)$.

IR (neat): $v_{\max } / \mathrm{cm}^{-1} 3070,3018,2957,2923,1661,1620,1448,1375,1357,1341,1301,1241$, $1170,1023,894,814$.

${ }^{1} \mathrm{H}$ NMR (300 MHz, $\left.\mathrm{CDCl}_{3}+\mathrm{CCl}_{4}\right): \delta 5.50-5.30(2 \mathrm{H}, \mathrm{m}), 4.78(1 \mathrm{H}, \mathrm{br} \mathrm{s}), 4.68(1 \mathrm{H}, \mathrm{br} \mathrm{s}), 2.85$ $(1 \mathrm{H}, \mathrm{dd}, \mathrm{J}=18.0$ and $6.6 \mathrm{~Hz}), 2.71(1 \mathrm{H}, \mathrm{d}$ of $\mathrm{t}, \mathrm{J}=12.6$ and $4.5 \mathrm{~Hz}), 2.65-2.45(4 \mathrm{H}, \mathrm{m}), 2.37(1$ $\mathrm{H}, \mathrm{dd}, \mathrm{J}=18.0$ and $2.4 \mathrm{~Hz}), 2.40-2.20(1 \mathrm{H}, \mathrm{m}), 1.98(1 \mathrm{H}, \mathrm{dd}, \mathrm{J}=15.0$ and $7.5 \mathrm{~Hz}), 1.79(3 \mathrm{H}, \mathrm{s})$ and $1.51(3 \mathrm{H}, \mathrm{s})$ [2 x olefinic $\left.\mathrm{CH}_{3}\right], 1.27\left(3 \mathrm{H}, \mathrm{s}\right.$, tert- $\left.\mathrm{CH}_{3}\right)$.

${ }^{13} \mathrm{C} \mathrm{NMR}\left(75 \mathrm{MHz}, \mathrm{CDCl}_{3}+\mathrm{CCl}_{4}\right): \delta 196.9(\mathrm{C}, \mathrm{C}=\mathrm{O}), 162.1(\mathrm{C}-7), 148.9\left(\mathrm{C}=\mathrm{CH}_{2}\right), 131.1(\mathrm{C}-8)$, 
127.8 and $127.3(\mathrm{C}-3$ and $\mathrm{C}-4), 115.1\left(\mathrm{C}=\mathrm{CH}_{2}\right), 54.2,47.3,39.4,36.8,29.7,28.7,27.7,21.7$, 10.8 .

Mass: m/z $230\left(\mathrm{M}^{+}, 5 \%\right), 215\left(\mathrm{M}-\mathrm{CH}_{3}, 5 \%\right), 161$ (10), 133 (12), 119 (12), 107 (10), 105 (10). HRMS: For $\mathrm{C}_{16} \mathrm{H}_{22} \mathrm{ONa}(\mathrm{M}+\mathrm{Na})$, Calcd.: 253.1568. Found: 253.1583 .

(+)-(1S,11S)-1,8-Dimethyl-11-isopropenylbicyclo[5.4.0]undeca-3,7-diene-9-one (12). $[\alpha]_{D}^{22}: 155.9\left(c 1.7, \mathrm{CHCl}_{3}\right)$.

$\mathrm{UV} / \mathrm{Vis}\left(\mathrm{CH}_{3} \mathrm{CN}\right): \lambda_{\max }=250.6(\epsilon=8808)$.

IR (neat): $v_{\max } / \mathrm{cm}^{-1} 3015,2960,1664,1456,1376,1326,1306,1056,896,822$.

${ }^{1} \mathrm{H}$ NMR (300 MHz, $\left.\mathrm{CDCl}_{3}+\mathrm{CCl}_{4}\right): \delta 5.60-5.40(2 \mathrm{H}, \mathrm{m}$, olefinic $\mathrm{H}), 4.98(1 \mathrm{H}, \mathrm{s})$ and $4.80(1 \mathrm{H}$, s) $\left[\mathrm{C}=\mathrm{CH}_{2}\right], 2.88(1 \mathrm{H}, \mathrm{dd}, \mathrm{J}=14.7$ and $4.2 \mathrm{~Hz}), 2.78(1 \mathrm{H}, \mathrm{d}$ of $\mathrm{t}, \mathrm{J}=12.9$ and $5.7 \mathrm{~Hz}), 2.69(1 \mathrm{H}$, dd, $\mathrm{J}=17.4$ and $14.4 \mathrm{~Hz}), 2.65-2.45(3 \mathrm{H}, \mathrm{m}), 2.40-2.20(1 \mathrm{H}, \mathrm{m}), 2.33(1 \mathrm{H}, \mathrm{dd}, \mathrm{J}=17.1$ and 3.9 $\mathrm{Hz}), 2.03(1 \mathrm{H}, \mathrm{dd}, \mathrm{J}=15.0$ and $9.0 \mathrm{~Hz}) 1.80(3 \mathrm{H}, \mathrm{s})$ and $1.77(3 \mathrm{H}, \mathrm{s})$ [2 x olefinic $\left.\mathrm{CH}_{3}\right], 1.09$ (3 $\mathrm{H}, \mathrm{s}$, tert $\left.-\mathrm{CH}_{3}\right)$.

${ }^{13} \mathrm{C}$ NMR $\left(75 \mathrm{MHz}, \mathrm{CDCl}_{3}+\mathrm{CCl}_{4}\right): \delta 198.2(\mathrm{C}, \mathrm{C}=\mathrm{O}), 165.0(\mathrm{C}, \mathrm{C}-7), 145.0\left(\mathrm{C}, C=C \mathrm{H}_{2}\right), 130.5$ $(\mathrm{C}, \mathrm{C}-8), 130.2(\mathrm{CH})$ and $125.4(\mathrm{CH})$ [C-3 and C-4], $115.2\left(\mathrm{CH}_{2}, \mathrm{C}=\mathrm{CH}_{2}\right), 47.9(\mathrm{C}, \mathrm{C}-1), 46.9$ (CH, C-11), $39.4\left(\mathrm{CH}_{2}\right), 33.7\left(\mathrm{CH}_{2}\right), 29.2\left(\mathrm{CH}_{2}\right), 26.6\left(\mathrm{CH}_{2}\right), 22.8\left(\mathrm{CH}_{3}\right), 21.5\left(\mathrm{CH}_{3}\right), 11.2\left(\mathrm{CH}_{3}\right)$. HRMS: For $\mathrm{C}_{16} \mathrm{H}_{22} \mathrm{ONa}(\mathrm{M}+\mathrm{Na})$, Calcd.: 231.1749. Found: 231.1768.

(+)-(4S,5R)-4-Allyl-3-(but-3-enyl)-5-isopropenyl-2-methylcyclohex-2-enone (13): $[\alpha]_{D}^{24}:+32.8\left(c 3.2, \mathrm{CHCl}_{3}\right)$.

$\mathrm{UV}\left(\mathrm{CH}_{3} \mathrm{CN}\right): \lambda_{\max }=249(\epsilon=11726)$.

IR (neat): $v_{\max } / \mathrm{cm}^{-1} 3077,2976,2928,1667,1643,1443,1416,1380,1358,1310,1226,1163$, 1083, 994, 913, 896.

${ }^{1} \mathrm{H}$ NMR (300 MHz, $\left.\mathrm{CDCl}_{3}+\mathrm{CCl}_{4}\right): \delta$ 5.90-5.65 $(2 \mathrm{H}, \mathrm{m}), 5.20-4.95(4 \mathrm{H}, \mathrm{m}), 4.79(1 \mathrm{H}, \mathrm{s}), 4.57$ $(1 \mathrm{H}, \mathrm{s}), 2.70-2.00(10 \mathrm{H}, \mathrm{m}), 1.75(3 \mathrm{H}, \mathrm{s})$ and $1.71(3 \mathrm{H}, \mathrm{s})$ [2 x olefinic $\left.\mathrm{CH}_{3}\right]$.

${ }^{13} \mathrm{C}$ NMR (75 MHz, $\left.\mathrm{CDCl}_{3}+\mathrm{CCl}_{4}\right): \delta 197.4(\mathrm{C}, \mathrm{C}=\mathrm{O}), 157.0(\mathrm{C}, \mathrm{C}-3), 145.9\left(\mathrm{C}, \mathrm{C}=\mathrm{CH}_{2}\right), 137.2$ $(\mathrm{CH}), 136.0(\mathrm{CH}), 131.4(\mathrm{C}, \mathrm{C}-2), 117.6\left(\mathrm{CH}_{2}\right), 115.6\left(\mathrm{CH}_{2}\right), 112.4\left(\mathrm{CH}_{2}\right), 42.0(\mathrm{CH}), 41.9(\mathrm{CH})$, $37.5\left(\mathrm{CH}_{2}\right), 36.6\left(\mathrm{CH}_{2}\right), 32.9\left(\mathrm{CH}_{2}\right), 31.6\left(\mathrm{CH}_{2}\right), 21.7\left(\mathrm{CH}_{3}\right), 10.9\left(\mathrm{CH}_{3}\right)$. HRMS: For $\mathrm{C}_{17} \mathrm{H}_{24} \mathrm{ONa}(\mathrm{M}+\mathrm{Na})$, Calcd.: 267.1725. Found: 267.1730. 
(-)-(1S,11R)-8-Methyl-11-isopropenylbicyclo[5.4.0]undeca-3,7-diene-9-one (14):

$[\alpha]_{D}^{24}:-66.5\left(c 1.7, \mathrm{CHCl}_{3}\right)$.

$\mathrm{UV} / \mathrm{Vis}\left(\mathrm{CH}_{3} \mathrm{CN}\right): \lambda_{\max }=246(\epsilon=12310)$.

IR (neat): $v_{\max } / \mathrm{cm}^{-1} 3072,3016,1667,1618,1443,1378,1359,1327,1305,1264,1112,894$, $669,644$.

${ }^{1} \mathrm{H}$ NMR (300 MHz, $\left.\mathrm{CDCl}_{3}+\mathrm{CCl}_{4}\right): \delta$ 5.65-5.50 $(2 \mathrm{H}, \mathrm{m}, \mathrm{H}-3$ and H-4), $4.82(1 \mathrm{H}, \mathrm{br}$ s) and 4.79 $(1 \mathrm{H}, \mathrm{s})\left[\mathrm{C}=\mathrm{CH}_{2}\right], 2.85-2.35(8 \mathrm{H}, \mathrm{m}), 2.37-2.19(1 \mathrm{H}, \mathrm{m}), 2.18-2.00(1 \mathrm{H}, \mathrm{m}), 1.79(3 \mathrm{H}, \mathrm{s})$ and $1.69(3 \mathrm{H}, \mathrm{s})\left[2 \mathrm{x}\right.$ olefinic $\left.\mathrm{CH}_{3}\right]$.

${ }^{13} \mathrm{C}$ NMR $\left(75 \mathrm{MHz}, \mathrm{CDCl}_{3}+\mathrm{CCl}_{4}\right): \delta 197.8(\mathrm{C}, \mathrm{C}=\mathrm{O}), 160.6(\mathrm{C}, \mathrm{C}-7), 145.6\left(\mathrm{C}, \mathrm{C}=\mathrm{CH}_{2}\right), 130.7$ $(\mathrm{C}, \mathrm{C}-8), 130.2(\mathrm{CH}), 127.0(\mathrm{CH}, \mathrm{C}-3$ and $\mathrm{C}-4), 113.5\left(\mathrm{CH}_{2}, \mathrm{C}=\mathrm{CH}_{2}\right), 48.0(\mathrm{CH}), 44.7(\mathrm{CH}), 42.3$ $\left(\mathrm{CH}_{2}\right), 30.4\left(\mathrm{CH}_{2}\right), 28.4\left(\mathrm{CH}_{2}\right), 28.3\left(\mathrm{CH}_{2}\right), 18.9\left(\mathrm{CH}_{3}\right), 11.0\left(\mathrm{CH}_{3}\right)$.

HRMS: For $\mathrm{C}_{15} \mathrm{H}_{20} \mathrm{ONa}(\mathrm{M}+\mathrm{Na})$, Calcd.: 239.1412. Found: 239.1422 .

\section{(-)-(1S,4S,5R)-4-Allyl-3-(but-3-enyl)-2-methyl-5-isopropenylcyclohex-2-en-1-ol (16):} $[\alpha]_{D}^{24}:-13.8\left(c 1.3, \mathrm{CHCl}_{3}\right)$.

IR (neat): $v_{\max } / \mathrm{cm}^{-1} 3345,3074,2976,2929,2861,1639,1439,1375,1025,993,910,892$. ${ }^{1} \mathrm{H}$ NMR (300 MHz, $\left.\mathrm{CDCl}_{3}+\mathrm{CCl}_{4}\right): \delta$ 5.90-5.77 $(1 \mathrm{H}, \mathrm{m}), 5.73-5.52(1 \mathrm{H}, \mathrm{m}), 5.10-4.92(4 \mathrm{H}, \mathrm{m})$, $4.79(1 \mathrm{H}, \mathrm{s}), 4.76(1 \mathrm{H}, \mathrm{s}), 3.85(1 \mathrm{H}, \mathrm{m}), 2.45-2.00(8 \mathrm{H}, \mathrm{m}), 2.00-1.65(3 \mathrm{H}, \mathrm{m}), 1.80(3 \mathrm{H}, \mathrm{s})$ and $1.76(3 \mathrm{H}, \mathrm{s})$ [2 x olefinic $\left.\mathrm{CH}_{3}\right]$.

${ }^{13} \mathrm{C}$ NMR (75 MHz, $\left.\mathrm{CDCl}_{3}+\mathrm{CCl}_{4}\right): \delta 150.2(\mathrm{C}), 138.4(\mathrm{CH}), 136.7(\mathrm{CH}), 134.4(\mathrm{C}), 131.1(\mathrm{C})$, $116.7\left(\mathrm{CH}_{2}\right), 114.8\left(\mathrm{CH}_{2}\right), 111.3\left(\mathrm{CH}_{2}\right), 70.4(\mathrm{CH}, \mathrm{C}-1), 40.9(\mathrm{CH}), 40.2(\mathrm{CH}), 35.7\left(\mathrm{CH}_{2}\right), 33.3$ $\left(\mathrm{CH}_{2}\right), 32.8\left(\mathrm{CH}_{2}\right), 30.5\left(\mathrm{CH}_{2}\right), 21.5\left(\mathrm{CH}_{3}\right), 15.9\left(\mathrm{CH}_{3}\right)$.

HRMS: For $\mathrm{C}_{17} \mathrm{H}_{26} \mathrm{ONa}(\mathrm{M}+\mathrm{Na})$, Calcd.: 269.1881. Found: 269.1898 .

(+)-(1S,2S,4R,5S,6R)-5-Allyl-6-(but-3-enyl)-1-methyl-4-isopropenyl-7oxabicyclo[4.1.0]heptane-2-ol (17):

$[\alpha]_{D}^{24}:+21.7\left(c 1.2, \mathrm{CHCl}_{3}\right)$.

IR (neat): $\nu_{\max } / \mathrm{cm}^{-1} 3423,3075,2976,2930,1642,1437,1379,1072,1037,912,869$.

${ }^{1} \mathrm{H}$ NMR $\left(300 \mathrm{MHz}, \mathrm{CDCl}_{3}+\mathrm{CCl}_{4}\right): \delta 5.82(1 \mathrm{H}, \mathrm{t}$ of dd, $\mathrm{J}=16.8,10.5$ and $6.6 \mathrm{~Hz}), 5.64-5.57(1$ H, m), 5.15-4.90 (4 H, m), $4.71(1 \mathrm{H} \mathrm{s}), 4.69(1 \mathrm{H}, \mathrm{s}), 3.67(1 \mathrm{H}, \mathrm{m}), 2.35-1.90(8 \mathrm{H}, \mathrm{m}), 1.72-$ 
$1.41(3 \mathrm{H}, \mathrm{m}), 1.64\left(3 \mathrm{H}, \mathrm{s}\right.$, olefinic $\left.\mathrm{CH}_{3}\right), 1.45\left(3 \mathrm{H}, \mathrm{s}\right.$, tert- $\left.\mathrm{CH}_{3}\right)$.

${ }^{13} \mathrm{C} \mathrm{NMR}\left(75 \mathrm{MHz}, \mathrm{CDCl}_{3}+\mathrm{CCl}_{4}\right): \delta 147.1\left(\mathrm{C}, \mathrm{C}=\mathrm{CH}_{2}\right), 138.1(\mathrm{CH}), 135.1(\mathrm{CH}), 118.4\left(\mathrm{CH}_{2}\right)$, $114.8\left(\mathrm{CH}_{2}\right), 112.0\left(\mathrm{CH}_{2}\right), 72.0(\mathrm{CH}, \mathrm{C}-2), 68.5(\mathrm{C}), 67.1(\mathrm{C}), 44.0(\mathrm{CH}), 35.9(\mathrm{CH}), 34.2\left(\mathrm{CH}_{2}\right)$, $33.3\left(\mathrm{CH}_{2}\right), 30.6\left(\mathrm{CH}_{2}\right), 29.4\left(\mathrm{CH}_{2}\right), 18.7\left(\mathrm{CH}_{3}\right), 14.5\left(\mathrm{CH}_{3}\right)$.

(-)-(1R,4S,5S,6R)-5-Allyl-6-(but-3-enyl)-1-methyl-4-isopropenyl-7-oxabicyclo[4.1.0]heptan2-one (15):

$[\alpha]_{D}^{23}:-2.6\left(c 2.7, \mathrm{CHCl}_{3}\right)$

IR (neat): $v_{\max } / \mathrm{cm}^{-1} 3076,2976,2933,1715,1642,1445,1378,1307,1108,994,914$.

${ }^{1} \mathrm{H}$ NMR (300 MHz, $\left.\mathrm{CDCl}_{3}+\mathrm{CCl}_{4}\right): \delta 5.83(1 \mathrm{H}, \mathrm{t}$ of dd, $\mathrm{J}=16.8,9.9$ and $6.6 \mathrm{~Hz}), 5.61(1 \mathrm{H}, \mathrm{t}$ of dd, $\mathrm{J}=16.8,10.2$ and $6.6 \mathrm{~Hz}), 5.15-4.95(4 \mathrm{H}, \mathrm{m}), 4.77(2 \mathrm{H}, \mathrm{s}), 2.93(1 \mathrm{H}, \mathrm{dd}, \mathrm{J}=13.5$ and 10.2 Hz), 2.60-2.50 (1 H, m), 2.40-2.20 (3 H, m), 2.20-1.96 (4 H, m), 1.75 (3 H, s, olefinic $\left.\mathrm{CH}_{3}\right)$, 1.54$1.40(1 \mathrm{H}, \mathrm{m}), 1.40\left(3 \mathrm{H}, \mathrm{s}\right.$, tert- $\left.\mathrm{CH}_{3}\right)$.

${ }^{13} \mathrm{C}$ NMR (75 MHz, $\left.\mathrm{CDCl}_{3}+\mathrm{CCl}_{4}\right): \delta 209.6(\mathrm{C}, \mathrm{C}=\mathrm{O}), 146.9\left(\mathrm{C}, \mathrm{C}=\mathrm{CH}_{2}\right), 137.5(\mathrm{CH}), 134.5$

$(\mathrm{CH}), 118.7\left(\mathrm{CH}_{2}\right), 115.5\left(\mathrm{CH}_{2}\right), 112.1\left(\mathrm{CH}_{2}\right), 73.2(\mathrm{C}), 65.8(\mathrm{C}), 45.5(\mathrm{CH}), 38.8\left(\mathrm{CH}_{2}\right), 37.5$ $(\mathrm{CH}), 35.7\left(\mathrm{CH}_{2}\right), 30.8\left(\mathrm{CH}_{2}\right), 29.3\left(\mathrm{CH}_{2}\right), 19.5\left(\mathrm{CH}_{3}\right), 11.4\left(\mathrm{CH}_{3}\right)$.

HRMS: For $\mathrm{C}_{17} \mathrm{H}_{24} \mathrm{O}_{2} \mathrm{Na}(\mathrm{M}+\mathrm{Na})$, Calcd.: 283.1674 Found: 283.1671.

\section{(-)-Methyl (1R,2R,3S,4R,5R)-4-Allyl-3-(but-3-enyl)-3-hydroxy-2-methyl-5-}

isopropenylcyclopentanecarboxylate (18):

$[\alpha]_{D}^{23}:-38.5\left(c 2.0, \mathrm{CHCl}_{3}\right)$.

IR (neat): $v_{\max } / \mathrm{cm}^{-1} 3513,3075,2950,1736,1640,1437,1376,1273,1196,1176,1022,996$, 909.

${ }^{1} \mathrm{H}$ NMR (300 MHz, $\left.\mathrm{CDCl}_{3}+\mathrm{CCl}_{4}\right)$ : $85.90-5.72(2 \mathrm{H}, \mathrm{m}), 5.10-4.75(6 \mathrm{H}, \mathrm{m}), 3.60(3 \mathrm{H}, \mathrm{s}$, $\left.\mathrm{COOCH}_{3}\right), 2.80-2.75(1 \mathrm{H}, \mathrm{m}), 2.71(1 \mathrm{H}, \mathrm{dd}, \mathrm{J}=10.5$ and $8.4 \mathrm{~Hz}), 2.53(1 \mathrm{H}, \mathrm{t}, \mathrm{J}=10.5 \mathrm{~Hz})$, $2.42(1 \mathrm{H}$, quintet, $\mathrm{J}=7.5 \mathrm{~Hz}), 2.33-2.10(4 \mathrm{H}, \mathrm{m}), 2.20(1 \mathrm{H}, \mathrm{d}$ of q, $\mathrm{J}=6.6$ and $6.6 \mathrm{~Hz}), 1.70$ (3 $\mathrm{H}$, s, olefinic $\left.\mathrm{CH}_{3}\right), 1.73-1.40(2 \mathrm{H}, \mathrm{m}), 1.00(3 \mathrm{H}, \mathrm{d}, \mathrm{J}=7.2 \mathrm{~Hz})$.

${ }^{13} \mathrm{C} \mathrm{NMR}\left(75 \mathrm{MHz}, \mathrm{CDCl}_{3}+\mathrm{CCl}_{4}\right): \delta 174.7(\mathrm{C}), 144.6\left(\mathrm{C}, \mathrm{C}=\mathrm{CH}_{2}\right), 139.1(\mathrm{CH}), 137.6(\mathrm{CH})$, $115.8\left(\mathrm{CH}_{2}\right), 114.5\left(\mathrm{CH}_{2}\right), 113.0\left(\mathrm{CH}_{2}\right), 81.7(\mathrm{C}), 54.5\left(\mathrm{CH}_{3}\right), 53.2(\mathrm{CH}), 51.5(\mathrm{CH}), 51.2(\mathrm{CH})$, $47.6(\mathrm{CH}), 35.1\left(\mathrm{CH}_{2}\right), 31.2\left(\mathrm{CH}_{2}\right), 28.7\left(\mathrm{CH}_{2}\right), 23.2\left(\mathrm{CH}_{3}\right), 14.6\left(\mathrm{CH}_{3}\right)$, 
HRMS: For $\mathrm{C}_{18} \mathrm{H}_{28} \mathrm{O}_{3} \mathrm{Na}(\mathrm{M}+\mathrm{Na})$, Calcd.: 315.1936 Found: 315.1936 .

(-)-Methyl $(1 R, 7 S, 8 S, 9 R, 10 R)$-1-hydroxy-10-methyl-8-isopropenylbicyclo[5.3.0]dec-4-ene-9carboxylate (19).

$[\alpha]_{D}^{22}:-67.3\left(c 1.1, \mathrm{CHCl}_{3}\right)$.

IR (neat): $v_{\max } / \mathrm{cm}^{-1} 3456,3077,3023,2934,2850,1732,1647,1454,1435,1374,1301,1262$, 1193, 1174, 1088, 1025, 971, 893, 832, 751.

${ }^{1} \mathrm{H}$ NMR (300 MHz, $\left.\mathrm{CDCl}_{3}+\mathrm{CCl}_{4}\right): \delta$ 5.90-5.78 $(1 \mathrm{H}, \mathrm{m}), 5.60-5.47(1 \mathrm{H}, \mathrm{m}), 4.87(1 \mathrm{H}, \mathrm{s}), 4.73$ $(1 \mathrm{H}, \mathrm{s}), 3.59\left(3 \mathrm{H}, \mathrm{s}, \mathrm{COOCH}_{3}\right), 2.75-2.25(6 \mathrm{H}, \mathrm{m}), 2.20-1.95(3 \mathrm{H}, \mathrm{m}), 1.69(3 \mathrm{H}, \mathrm{s}$, olefinic $\left.\mathrm{CH}_{3}\right), 1.80-1.40(2 \mathrm{H}, \mathrm{m}), 0.92\left(3 \mathrm{H}, \mathrm{d}, \mathrm{J}=6.9 \mathrm{~Hz}, \mathrm{sec}-\mathrm{CH}_{3}\right)$.

${ }^{13} \mathrm{C}$ NMR (75 MHz, $\left.\mathrm{CDCl}_{3}+\mathrm{CCl}_{4}\right): \delta 174.9(\mathrm{C}), 143.5\left(\mathrm{C}, \mathrm{C}=\mathrm{CH}_{2}\right), 133.3(\mathrm{CH}), 128.0(\mathrm{CH})$, 112.6 $\left(\mathrm{CH}_{2}, \mathrm{C}=\mathrm{CH}_{2}\right), 81.4(\mathrm{C}), 52.7\left(\mathrm{CH}_{3}\right), 51.4(\mathrm{CH}), 50.7(\mathrm{CH}), 48.8(\mathrm{CH}), 47.9(\mathrm{CH}), 29.3$ $\left(\mathrm{CH}_{2}\right), 25.1\left(\mathrm{CH}_{2}\right), 22.9\left(\mathrm{CH}_{3}\right), 22.4\left(\mathrm{CH}_{2}\right), 13.1\left(\mathrm{CH}_{3}\right)$.

HRMS: For $\mathrm{C}_{16} \mathrm{H}_{24} \mathrm{O}_{3} \mathrm{Na}(\mathrm{M}+\mathrm{Na})$, Calcd.: 287.1623. Found: 287.1637.

(+)-(1S,4S,5S)-4-Allyl-3-(but-3-enyl)-2,4-dimethyl-5-isopropenylcyclohex-2-ene-1-ol: (Obtained by reduction of $\mathbf{1 0}$ ) $[\alpha]_{D}^{22}:+98.4\left(c 5.0, \mathrm{CHCl}_{3}\right)$.

IR (neat): $v_{\max } / \mathrm{cm}^{-1} 3282,3073,2968,2935,2874,1639,1468,1447,1376,1308,1117,1065$, 1020, 997, 911, 898 .

${ }^{1} \mathrm{H}$ NMR (300 MHz, $\left.\mathrm{CDCl}_{3}+\mathrm{CCl}_{4}\right):$ 5 5.84-5.73 $(1 \mathrm{H}, \mathrm{m}), 5.50-5.40(1 \mathrm{H}, \mathrm{m}), 5.03-4.89(5 \mathrm{H}, \mathrm{m})$, $4.71(1 \mathrm{H}, \mathrm{s}), 4.00(1 \mathrm{H}, \mathrm{dd}, \mathrm{J}=8.4$ and $6.6 \mathrm{~Hz}), 2.40-1.93(6 \mathrm{H}, \mathrm{m}), 1.84(1 \mathrm{H}, \mathrm{ddd}, \mathrm{J}=12.0,6.0$ and $2.7 \mathrm{~Hz}), 1.80-1.73(1 \mathrm{H}, \mathrm{m}), 1.70-1.50(2 \mathrm{H}, \mathrm{m}), 1.73\left(6 \mathrm{H}, \mathrm{s}, 2\right.$ x olefinic $\left.\mathrm{CH}_{3}\right), 0.93(3 \mathrm{H}, \mathrm{s}$, tert- $\left.\mathrm{CH}_{3}\right)$.

${ }^{13} \mathrm{C} \mathrm{NMR}\left(75 \mathrm{MHz}, \mathrm{CDCl}_{3}+\mathrm{CCl}_{4}\right): \delta 146.4(\mathrm{C}), 138.7(\mathrm{C}), 138.6(\mathrm{CH}), 135.8(\mathrm{CH}), 133.2(\mathrm{C})$, 117.2 $\left(\mathrm{CH}_{2}\right), 114.9\left(\mathrm{CH}_{2}\right), 114.3\left(\mathrm{CH}_{2}\right), 71.4(\mathrm{CH}), 44.1(\mathrm{CH}), 43.5(\mathrm{C}), 41.5\left(\mathrm{CH}_{2}\right), 34.4\left(\mathrm{CH}_{2}\right)$, $34.3\left(\mathrm{CH}_{2}\right), 28.5\left(\mathrm{CH}_{2}\right), 22.7\left(\mathrm{CH}_{3}\right), 22.7\left(\mathrm{CH}_{3}\right), 15.5\left(\mathrm{CH}_{3}\right)$.

HRMS: For $\mathrm{C}_{18} \mathrm{H}_{28} \mathrm{ONa}(\mathrm{M}+\mathrm{Na})$, Calcd.: 283.2038. Found: 283.2023.

(+)-(1S,2S,4S,5S,6R)-5-Allyl-6-(but-3-enyl)-1,5-dimethyl-4-isopropenyl-7- 
oxabicyclo[4.1.0]heptane-2-ol:

$[\alpha]_{D}^{22}:+39.7\left(c 6.0, \mathrm{CHCl}_{3}\right)$.

IR (neat): $v_{\max } / \mathrm{cm}^{-1} 3426,3074,2977,1641,1443,1375,1035,1026,911$.

${ }^{1} \mathrm{H}$ NMR $\left(300 \mathrm{MHz}, \mathrm{CDCl}_{3}+\mathrm{CCl}_{4}\right): \delta 5.84-5.70(1 \mathrm{H}, \mathrm{t}$ of dd, $\mathrm{J}=17.1,9.3$ and $6.5 \mathrm{~Hz}), 5.69-5.56$ (1 H, m), 5.20-4.90 (4 H, m), $4.84(1 \mathrm{H}, \mathrm{s}), 4.66(1 \mathrm{H}, \mathrm{s}), 3.63(1 \mathrm{H}, \mathrm{br} \mathrm{s}), 2.39(1 \mathrm{H}, \mathrm{dd}, \mathrm{J}=15.9$ and 8.4 Hz), 2.27-1.90 (5 H, m), 1.85-1.62 (2 H, m), $1.69\left(3 \mathrm{H}\right.$, s, olefinic $\left.\mathrm{CH}_{3}\right), 1.51-1.39(2 \mathrm{H}$, m), $1.41(3 \mathrm{H}, \mathrm{s}), 0.95\left(3 \mathrm{H}, \mathrm{s}\right.$, tert $\left.\mathrm{CH}_{3}\right)$.

${ }^{13} \mathrm{C}$ NMR $\left(75 \mathrm{MHz}, \mathrm{CDCl}_{3}+\mathrm{CCl}_{4}\right): \delta 145.6(\mathrm{C}), 138.4(\mathrm{CH}), 135.3(\mathrm{CH}), 118.6\left(\mathrm{CH}_{2}\right), 115.0$ $\left(\mathrm{CH}_{2}\right), 114.7\left(\mathrm{CH}_{2}\right), 72.7(\mathrm{CH}), 71.0(\mathrm{C}), 67.0(\mathrm{C}), 45.3(\mathrm{CH}, \mathrm{C}-4), 41.7\left(\mathrm{CH}_{2}\right), 40.6(\mathrm{C}), 31.6$ $\left(\mathrm{CH}_{2}\right), 31.2\left(\mathrm{CH}_{2}\right), 28.7\left(\mathrm{CH}_{2}\right), 21.8\left(\mathrm{CH}_{3}\right), 20.0\left(\mathrm{CH}_{3}\right), 14.9\left(\mathrm{CH}_{3}\right)$.

HRMS: For $\mathrm{C}_{18} \mathrm{H}_{28} \mathrm{O}_{2} \mathrm{Na}(\mathrm{M}+\mathrm{Na})$, Calcd.: 299.1987. Found: 299.1974.

\section{(+)-(1R,4S,5S,6R)-5-Allyl-6-(but-3-enyl)-1,5-dimethyl-4-isopropenyl-7-}

oxabicyclo[4.1.0]heptane-2-one (20)

$[\alpha]_{D}^{24}: 7.5\left(c 2.0, \mathrm{CHCl}_{3}\right)$.

IR (neat): $v_{\text {max }} / \mathrm{cm}^{-1} 3076,2976,2933,1717(\mathrm{C}=\mathrm{O}), 1638(\mathrm{C}=\mathrm{C}), 1457,1376,1315,1032,994$, $913\left(\mathrm{C}=\mathrm{CH}_{2}\right), 863$.

${ }^{1} \mathrm{H}$ NMR (300 MHz, $\left.\mathrm{CDCl}_{3}+\mathrm{CCl}_{4}\right): \delta$ 5.81-5.50 (2 H, m), 5.16-4.90 (4 H, m), $4.84(1 \mathrm{H}, \mathrm{s}), 4.71$ $(1 \mathrm{H}, \mathrm{s}), 2.98(1 \mathrm{H}, \mathrm{dd}, \mathrm{J}=12.9$ and $11.5 \mathrm{~Hz}), 2.66(1 \mathrm{H}, \mathrm{dd}, \mathrm{J}=11.5$ and $6.0 \mathrm{~Hz}), 2.30(1 \mathrm{H}, \mathrm{dd}, \mathrm{J}$ $=15.0$ and $7.5 \mathrm{~Hz}), 2.20-1.90(5 \mathrm{H}, \mathrm{m}), 1.80-1.65(1 \mathrm{H}, \mathrm{m}), 1.78\left(3 \mathrm{H}\right.$, s, olefinic $\left.\mathrm{CH}_{3}\right), 1.36(3 \mathrm{H}$, s), $1.10\left(3 \mathrm{H}, \mathrm{s}\right.$, tert $\left.\mathrm{CH}_{3}\right)$.

${ }^{13} \mathrm{C}$ NMR (75 MHz, $\left.\mathrm{CDCl}_{3}+\mathrm{CCl}_{4}\right): \delta 208.7(\mathrm{C}, \mathrm{C}=\mathrm{O}), 145.2(\mathrm{C}), 137.8(\mathrm{CH}), 133.8(\mathrm{CH}), 119.5$ $\left(\mathrm{CH}_{2}\right), 115.9\left(\mathrm{CH}_{2}\right), 115.2\left(\mathrm{CH}_{2}\right), 75.3(\mathrm{CH}, \mathrm{C}-1), 65.9(\mathrm{C}, \mathrm{C}-6), 49.5(\mathrm{CH}, \mathrm{C}-4), 43.0\left(\mathrm{CH}_{2}\right)$, $41.9(\mathrm{C}, \mathrm{C}-5), 38.4\left(\mathrm{CH}_{2}\right), 30.7\left(\mathrm{CH}_{2}\right), 28.4\left(\mathrm{CH}_{2}\right), 21.4\left(\mathrm{CH}_{3}\right), 20.4\left(\mathrm{CH}_{3}\right), 11.9\left(\mathrm{CH}_{3}\right)$. HRMS: For $\mathrm{C}_{18} \mathrm{H}_{26} \mathrm{O}_{2} \mathrm{Na}(\mathrm{M}+\mathrm{Na})$, Calcd.: 297.1830. Found: 297.1834.

\section{(-)-Methyl (1R,2R,3S,4S,5R)-4-Allyl-3-(but-3-enyl)-3-hydroxy-2,4-dimethyl-5-} isopropenylcyclopentanecarboxylate (21):

$[\alpha]_{D}^{21}:-82.0\left(\right.$ c 2.0, $\left.\mathrm{CHCl}_{3}\right)$.

IR (neat): $v_{\max } / \mathrm{cm}^{-1} 3560(\mathrm{OH}), 3075,2975,1735(\mathrm{O}-\mathrm{C}=\mathrm{O}), 1638(\mathrm{C}=\mathrm{C}), 1435,1379,1201$, 
1176, 1028, 996, $910\left(\mathrm{C}=\mathrm{CH}_{2}\right)$.

${ }^{1} \mathrm{H}$ NMR (300 MHz, $\left.\mathrm{CDCl}_{3}+\mathrm{CCl}_{4}\right):$ 5 5.90-5.75 (2 H, m), 5.15-4.85 (5 H, m), $4.84(1 \mathrm{H}, \mathrm{s}), 3.59$

( $3 \mathrm{H}, \mathrm{s}, \mathrm{COOMe}), 2.80-2.60(3 \mathrm{H}, \mathrm{m}), 2.38-2.12(3 \mathrm{H}, \mathrm{m}), 2.08(1 \mathrm{H}, \mathrm{dd}, \mathrm{J}=14.1$ and $6.3 \mathrm{~Hz})$, $1.96(1 \mathrm{H}, \mathrm{dd}, \mathrm{J}=14.1$ and $8.7 \mathrm{~Hz}), 1.76\left(3 \mathrm{H}\right.$, s, olefinic $\left.\mathrm{CH}_{3}\right), 1.65-1.37(2 \mathrm{H}, \mathrm{m}), 1.15(3 \mathrm{H}, \mathrm{d}$, $\mathrm{J}=6.6 \mathrm{~Hz}), 1.03\left(3 \mathrm{H}, \mathrm{s}\right.$, tert $\left.-\mathrm{CH}_{3}\right)$.

${ }^{13} \mathrm{C}$ NMR $\left(75 \mathrm{MHz}, \mathrm{CDCl}_{3}+\mathrm{CCl}_{4}\right): \delta 174.0(\mathrm{C}), 148.1(\mathrm{C}), 139.4(\mathrm{CH}), 134.8(\mathrm{CH}), 118.2\left(\mathrm{CH}_{2}\right)$, $114.4\left(\mathrm{CH}_{2}\right), 113.6\left(\mathrm{CH}_{2}\right), 85.5(\mathrm{C}), 54.1\left(\mathrm{CH}_{3}\right), 53.7(\mathrm{C}), 53.2(\mathrm{CH}), 51.5(\mathrm{CH}), 47.1(\mathrm{CH}), 41.8$ $\left(\mathrm{CH}_{2}\right), 31.7\left(\mathrm{CH}_{2}\right), 28.7\left(\mathrm{CH}_{2}\right), 27.0\left(\mathrm{CH}_{3}\right), 19.2\left(\mathrm{CH}_{3}\right), 17.8\left(\mathrm{CH}_{3}\right)$.

HRMS: For $\mathrm{C}_{19} \mathrm{H}_{30} \mathrm{O}_{3} \mathrm{Na}(\mathrm{M}+\mathrm{Na})$, Calcd.: 329.2093. Found: 329.2084 .

\section{(-)-Methyl (1S,7S,8S,9R,10R)-1-hydroxy-7,10-dimethyl-8-isopropenylbicyclo[5.3.0]dec-4-} ene-9-carboxylate (22):

$[\alpha]_{D}^{22}:-25.0\left(c 1.0, \mathrm{CHCl}_{3}\right)$.

IR (neat): $v_{\max } / \mathrm{cm}^{-1} 3532,3071,1730,1644,1632,1435,1374,1272,1176,1109,1023,895$, 733.

${ }^{1} \mathrm{H}$ NMR $\left(300 \mathrm{MHz}, \mathrm{CDCl}_{3}+\mathrm{CCl}_{4}\right): \delta$ 5.83-5.77 (1 H, m), 5.58-5.51 (1 H, m), $4.91(1 \mathrm{H}, \mathrm{s}), 4.74$ $(1 \mathrm{H}, \mathrm{s}), 3.63\left(3 \mathrm{H}, \mathrm{s}, \mathrm{COOCH}_{3}\right), 2.97(1 \mathrm{H}, \mathrm{d}, \mathrm{J}=11.7 \mathrm{~Hz}), 2.71-2.60(2 \mathrm{H}, \mathrm{m}), 2.44$ (1 H, br t, J $=14.0 \mathrm{~Hz}), 2.20(2 \mathrm{H}, \mathrm{d}, \mathrm{J}=6.3 \mathrm{~Hz}), 2.15-2.05(1 \mathrm{H}, \mathrm{m}), 1.80-1.76(1 \mathrm{H}, \mathrm{m}), 1.76(3 \mathrm{H}, \mathrm{s}$, olefinic $\left.\mathrm{CH}_{3}\right), 1.72(1 \mathrm{H}, \mathrm{dd}, \mathrm{J}=11.7$ and $2.7 \mathrm{~Hz}), 1.65(1 \mathrm{H}, \mathrm{ddd}, \mathrm{J}=14.3,6.0$ and $3.0 \mathrm{~Hz}), 1.07$ $\left(3 \mathrm{H}, \mathrm{s}\right.$, tert $\left.-\mathrm{CH}_{3}\right), 0.97\left(3 \mathrm{H}, \mathrm{d}, \mathrm{J}=6.4 \mathrm{~Hz}\right.$, sec $\left.-\mathrm{CH}_{3}\right)$.

${ }^{13} \mathrm{C} \mathrm{NMR}\left(75 \mathrm{MHz}, \mathrm{CDCl}_{3}+\mathrm{CCl}_{4}\right): \delta 174.4(\mathrm{C}), 143.6\left(\mathrm{C}, C=\mathrm{CH}_{2}\right), 132.7(\mathrm{CH}), 128.1(\mathrm{CH})$, $115.0\left(\mathrm{CH}_{2}, \mathrm{C}=\mathrm{CH}_{2}\right), 82.9(\mathrm{C}, \mathrm{C}-1), 54.1\left(\mathrm{CH}_{3}\right), 51.8(\mathrm{CH}), 51.4(\mathrm{CH}), 50.9(\mathrm{C}), 46.7(\mathrm{CH}), 37.9$ $\left(\mathrm{CH}_{2}\right), 31.2\left(\mathrm{CH}_{2}\right), 25.5\left(\mathrm{CH}_{3}\right), 23.3\left(\mathrm{CH}_{2}\right), 20.5\left(\mathrm{CH}_{3}\right), 13.8\left(\mathrm{CH}_{3}\right)$. HRMS: For $\mathrm{C}_{17} \mathrm{H}_{26} \mathrm{O}_{3} \mathrm{Na}(\mathrm{M}+\mathrm{Na})$, Calcd.: 301.1780. Found: 301.1771.

(+)-(1S,4R,5S)-4-Allyl-3-(but-3-enyl)-2,4-dimethyl-5-isopropenylcyclohex-2-en-1-ol: (Obtained by reduction of 9 )

$[\alpha]_{D}^{22}:+78.5\left(c 5.3, \mathrm{CHCl}_{3}\right)$.

IR (neat): $v_{\max } / \mathrm{cm}^{-1} 3352,3075,2975,2940,2875,1638,1142,1375,1077,1019,1003,908$. ${ }^{1} \mathrm{H}$ NMR (300 MHz, $\left.\mathrm{CDCl}_{3}+\mathrm{CCl}_{4}\right): \delta$ 5.90-5.65 (2 H, m), 5.05-4.90 (5 H, m), $4.81(1 \mathrm{H}, \mathrm{s}), 3.94$ 
(1 H, br s, CHOH), 2.40-2.04 (7 H, m), 2.05-1.85 (3 H, m), $1.81(3 \mathrm{H}, \mathrm{s})$ and $1.77(3 \mathrm{H}, \mathrm{s})[2 \mathrm{x}$ olefinic $\left.\mathrm{CH}_{3}\right) 1.05\left(3 \mathrm{H}\right.$, s, tert- $\left.\mathrm{CH}_{3}\right)$.

${ }^{13} \mathrm{C}$ NMR (75 MHz, $\left.\mathrm{CDCl}_{3}+\mathrm{CCl}_{4}\right): \delta 147.8(\mathrm{C}), 139.1(\mathrm{C}), 138.5(\mathrm{CH}), 136.6(\mathrm{CH}), 131.3(\mathrm{C})$, $116.2\left(\mathrm{CH}_{2}\right), 114.4\left(2 \mathrm{C}, \mathrm{CH}_{2}\right), 70.8(\mathrm{CH}), 49.3(\mathrm{CH}), 42.0(\mathrm{C}), 40.7\left(\mathrm{CH}_{2}\right), 34.0\left(\mathrm{CH}_{2}\right), 33.7$ $\left(\mathrm{CH}_{2}\right), 28.9\left(\mathrm{CH}_{2}\right), 26.5\left(\mathrm{CH}_{3}\right), 24.4\left(\mathrm{CH}_{3}\right), 15.8\left(\mathrm{CH}_{3}\right)$. Mass: m/e 242 (M- $\left.\mathrm{H}_{2} \mathrm{O}, 2 \%\right), 219$ (10), 201 (10), 177 (8), 159 (40), 149 (10), 147 (8), 145 (10), 135 (20), 133 (100), 123 (50), 121 (40), 119 (50), 107 (50), 105 (40).

HRMS: For $\mathrm{C}_{18} \mathrm{H}_{28} \mathrm{ONa}(\mathrm{M}+\mathrm{Na})$, Calcd.: 283.2038. Found: 283.2039.

\section{(+)-(1S,2S,4S,5R,6R)-5-Allyl-6-(but-3-enyl)-1,5-dimethyl-4-isopropenyl-7-} oxabicyclo[4.1.0]heptan-2-ol:

$[\alpha]_{D}^{22}: 114.0\left(c 4.0, \mathrm{CHCl}_{3}\right)$.

IR (neat): $v_{\max } / \mathrm{cm}^{-1} 3435,3075,2976,2932,1640,1445,1378,1033,910$.

${ }^{1} \mathrm{H}$ NMR (300 MHz, $\left.\mathrm{CDCl}_{3}+\mathrm{CCl}_{4}\right): \delta$ 6.00-5.60 (2 H, m), 5.15-4.85 (5 H, m), $4.65(1 \mathrm{H}, \mathrm{s}), 3.70$ $(1 \mathrm{H}, \mathrm{m}), 2.55(1 \mathrm{H}, \mathrm{dd}, \mathrm{J}=12.6$ and $6.6 \mathrm{~Hz}), 2.30-2.07(2 \mathrm{H}, \mathrm{m}), 1.85(1 \mathrm{H}, \mathrm{dd}, \mathrm{J}=12.0$ and 1.8 $\mathrm{Hz}), 1.78-1.24(6 \mathrm{H}, \mathrm{m}), 1.71\left(3 \mathrm{H}\right.$, s, olefinic $\left.\mathrm{CH}_{3}\right), 1.48(3 \mathrm{H}, \mathrm{s}), 1.18\left(3 \mathrm{H}, \mathrm{s}\right.$, tert- $\left.\mathrm{CH}_{3}\right)$.

${ }^{13} \mathrm{C}$ NMR $\left(75 \mathrm{MHz}, \mathrm{CDCl}_{3}+\mathrm{CCl}_{4}\right): \delta 145.3(\mathrm{C}), 137.7(\mathrm{CH}), 136.2(\mathrm{CH}), 117.6\left(\mathrm{CH}_{2}\right), 115.7$ $\left(\mathrm{CH}_{2}\right), 114.6\left(\mathrm{CH}_{2}\right), 73.2(\mathrm{CH}), 73.1(\mathrm{C}), 66.5(\mathrm{C}), 51.9(\mathrm{CH}), 40.3(\mathrm{C}), 39.5\left(\mathrm{CH}_{2}\right), 34.8\left(\mathrm{CH}_{2}\right)$, $31.7\left(\mathrm{CH}_{2}\right), 31.0\left(\mathrm{CH}_{2}\right), 23.4\left(\mathrm{CH}_{3}\right), 23.0\left(\mathrm{CH}_{3}\right), 16.4\left(\mathrm{CH}_{3}\right)$.

HRMS: For $\mathrm{C}_{18} \mathrm{H}_{28} \mathrm{O}_{2} \mathrm{Na}(\mathrm{M}+\mathrm{Na})$, Calcd.: 299.1987. Found: 299.1997.

\section{(+)-(1R,4S,5R,6R)-5-Allyl-6-(but-3-enyl)-1,5-dimethyl-4-isopropenyl-7-}

oxabicyclo[4.1.0]heptan-2-one:

$[\alpha]_{D}^{23}: 111.4\left(c 1.4, \mathrm{CHCl}_{3}\right)$.

IR (neat): $v_{\max } / \mathrm{cm}^{-1} 3076,2976,2933,1715,1639,1439,1380,1315,1085,998,912$. ${ }^{1} \mathrm{H}$ NMR (300 MHz, $\left.\mathrm{CDCl}_{3}+\mathrm{CCl}_{4}\right): \delta$ 6.05-5.85 $(1 \mathrm{H}, \mathrm{m}), 5.80-5.60(1 \mathrm{H}, \mathrm{m}), 5.14(1 \mathrm{H}, \mathrm{d}, \mathrm{J}=$ $18.1 \mathrm{~Hz}), 5.12(1 \mathrm{H}, \mathrm{d}, \mathrm{J}=9.9 \mathrm{~Hz}), 5.05-4.92(3 \mathrm{H}, \mathrm{m}), 4.74(1 \mathrm{H}, \mathrm{s}), 3.03(1 \mathrm{H}, \mathrm{t}, \mathrm{J}=13.5 \mathrm{~Hz})$, $2.65(1 \mathrm{H}, \mathrm{dd}, \mathrm{J}=12.9$ and $6.9 \mathrm{~Hz}), 2.36(1 \mathrm{H}, \mathrm{dd}, \mathrm{J}=14.1$ and $3.0 \mathrm{~Hz}), 2.30-2.03(3 \mathrm{H}, \mathrm{m}), 2.01$ $(1 \mathrm{H}, \mathrm{dd}, \mathrm{J}=13.5$ and $3.3 \mathrm{~Hz}), 1.92(1 \mathrm{H}, \mathrm{dd}, \mathrm{J}=12.9$ and $8.1 \mathrm{~Hz}), 1.75\left(3 \mathrm{H}\right.$, s, olefinic $\left.\mathrm{CH}_{3}\right)$, 1.50-1.36 (1 H, m), $1.41(3 \mathrm{H}, \mathrm{s}), 1.23\left(3 \mathrm{H}, \mathrm{s}\right.$, tert $\left.-\mathrm{CH}_{3}\right)$. 
${ }^{13} \mathrm{C}$ NMR (75 MHz, $\left.\mathrm{CDCl}_{3}+\mathrm{CCl}_{4}\right): \delta 208.5(\mathrm{C}, \mathrm{C}=\mathrm{O}), 144.0(\mathrm{C}), 137.3(\mathrm{CH}), 135.1(\mathrm{CH}), 118.4$ $\left(\mathrm{CH}_{2}\right), 116.6\left(\mathrm{CH}_{2}\right), 115.0\left(\mathrm{CH}_{2}\right), 76.7(\mathrm{C}), 64.9(\mathrm{C}), 55.3(\mathrm{CH}), 41.6(\mathrm{C}), 39.1\left(\mathrm{CH}_{2}\right), 38.4$ $\left(\mathrm{CH}_{2}\right), 34.4\left(\mathrm{CH}_{2}\right), 30.5\left(\mathrm{CH}_{2}\right), 23.3\left(\mathrm{CH}_{3}\right), 22.8\left(\mathrm{CH}_{3}\right), 12.9\left(\mathrm{CH}_{3}\right)$, HRMS: For $\mathrm{C}_{18} \mathrm{H}_{26} \mathrm{O}_{2} \mathrm{Na}(\mathrm{M}+\mathrm{Na})$, Calcd.: 297.1830. Found: 297.1824.

\section{(-)-Methyl (1R,2R,3S,4R,5R)-4-Allyl-3-(but-3-enyl)-3-hydroxy-2,4-dimethyl-5-}

\section{isopropenylcyclopentanecarboxylate:}

$[\alpha]_{D}^{24}:-66.9\left(c 1.3, \mathrm{CHCl}_{3}\right)$.

IR (neat): $v_{\max } / \mathrm{cm}^{-1} 3555(\mathrm{OH}), 3073,2974,1736(\mathrm{C}=\mathrm{O}), 1638(\mathrm{C}=\mathrm{C}), 1436,1384,1203,1029$, $909\left(\mathrm{C}=\mathrm{CH}_{2}\right)$.

${ }^{1} \mathrm{H}$ NMR (300 MHz, $\left.\mathrm{CDCl}_{3}+\mathrm{CCl}_{4}\right): \delta$ 5.94-5.75 (2 H, m), 5.08-4.90 (6 H, m), $3.62(3 \mathrm{H}, \mathrm{s}$, COOMe), $2.81(1 \mathrm{H}, \mathrm{dd}, \mathrm{J}=9.0$ and $9.0 \mathrm{~Hz}), 2.77-2.65(2 \mathrm{H}, \mathrm{m}), 2.41(1 \mathrm{H}, \mathrm{s}), 2.38-2.00(2 \mathrm{H}$, m), $2.23(2 \mathrm{H}, \mathrm{d}, \mathrm{J}=6.9 \mathrm{~Hz}), 1.83\left(3 \mathrm{H}, \mathrm{s}\right.$, olefinic $\left.\mathrm{CH}_{3}\right), 1.80-1.44(2 \mathrm{H}, \mathrm{m}), 1.14(3 \mathrm{H}, \mathrm{d}, \mathrm{J}=6.9$ $\mathrm{Hz}$, sec $\left.-\mathrm{CH}_{3}\right), 0.98\left(3 \mathrm{H}, \mathrm{s}\right.$, tert $\left.-\mathrm{CH}_{3}\right)$.

${ }^{13} \mathrm{C} \mathrm{NMR}\left(75 \mathrm{MHz}, \mathrm{CDCl}_{3}+\mathrm{CCl}_{4}\right): \delta 174.5(\mathrm{C}), 146.7(\mathrm{C}), 139.4(\mathrm{CH}), 136.3(\mathrm{CH}), 117.2\left(\mathrm{CH}_{2}\right)$, $115.3\left(\mathrm{CH}_{2}\right), 114.4\left(\mathrm{CH}_{2}\right), 85.8(\mathrm{C}), 59.3(\mathrm{CH}), 55.3\left(\mathrm{CH}_{3}\right), 53.1(\mathrm{C}), 51.5(\mathrm{CH}), 48.0(\mathrm{CH}), 39.0$ $\left(\mathrm{CH}_{2}\right), 33.1\left(\mathrm{CH}_{2}\right), 28.7\left(\mathrm{CH}_{2}\right), 26.6\left(\mathrm{CH}_{3}\right), 24.6\left(\mathrm{CH}_{3}\right), 19.5\left(\mathrm{CH}_{3}\right)$.

HRMS: For $\mathrm{C}_{19} \mathrm{H}_{30} \mathrm{O}_{3} \mathrm{Na}(\mathrm{M}+\mathrm{Na})$, Calcd.: 329.2093. Found: 329.2076.

(-)-Methyl (1S,7R,8R,9R,10R)-7,10-dimethyl-1-hydroxy-8-isopropenylbicyclo[5.3.0]dec-4ene-9-carboxylate (23):

$[\alpha]_{D}^{22}:-73.0\left(c 1.0, \mathrm{CHCl}_{3}\right)$.

IR (neat): $v_{\max } / \mathrm{cm}^{-1} 3500,2984,1718,1647,1455,1437,1378,1357,1265,1225,1198,1098$, 1040, 1030, 991, 928, 891, 709.

${ }^{1} \mathrm{H}$ NMR $\left(300 \mathrm{MHz}, \mathrm{CDCl}_{3}+\mathrm{CCl}_{4}\right): \delta$ 5.65-5.56 (1 H, m), 5.52-5.45 (1 H, m), $4.99(1 \mathrm{H}, \mathrm{s}), 4.93$ (1 H, s), 3.62 (3 H, s), 2.97 (1 H, t, J = 9.0 Hz), 2.88-2.73 (1 H, m), 2.73-2.60 (2 H, m), 2.60-2.30 (2 H, m), 2.15-2.00 (1 H, m), $1.94(1 \mathrm{H}, \mathrm{dd}, \mathrm{J}=16.2$ and $7.8 \mathrm{~Hz}), 1.83\left(3 \mathrm{H}, \mathrm{s}\right.$, olefinic $\left.\mathrm{CH}_{3}\right)$, 1.75-1.65 (2 H, m), $1.16\left(3 \mathrm{H}, \mathrm{s}\right.$, tert $\left.-\mathrm{CH}_{3}\right), 1.11\left(3 \mathrm{H}, \mathrm{d}, \mathrm{J}=7.5 \mathrm{~Hz}\right.$, sec $\left.-\mathrm{CH}_{3}\right)$.

${ }^{13} \mathrm{C} \mathrm{NMR}\left(75 \mathrm{MHz}, \mathrm{CDCl}_{3}+\mathrm{CCl}_{4}\right): \delta 174.1(\mathrm{C}), 148.7(\mathrm{C}), 129.8(\mathrm{CH}), 127.1(\mathrm{CH}), 113.6$ $\left(\mathrm{CH}_{2}\right), 85.7(\mathrm{C}), 60.0(\mathrm{CH}), 55.0\left(\mathrm{CH}_{3}\right), 53.5(\mathrm{C}), 51.5(\mathrm{CH}), 49.4(\mathrm{CH}), 34.9\left(\mathrm{CH}_{2}\right), 30.1\left(\mathrm{CH}_{2}\right)$, 
$27.4\left(\mathrm{CH}_{3}\right), 25.9\left(\mathrm{CH}_{3}\right), 23.7\left(\mathrm{CH}_{2}\right), 19.3\left(\mathrm{CH}_{3}\right)$.

HRMS: For $\mathrm{C}_{17} \mathrm{H}_{26} \mathrm{O}_{3} \mathrm{Na}(\mathrm{M}+\mathrm{Na})$, Calcd.: 301.1780. Found: 301.1779 .

(-)-(2S,3S,4S)-2-Acetyl-3-allyl-2-(but-3-enyl)-4-isopropenyl-3-methylcyclopentanone (24): $[\alpha]_{D}^{23}:-25.8\left(c 2.4, \mathrm{CHCl}_{3}\right)$.

IR (neat): $v_{\max } / \mathrm{cm}^{-1} 3077,2972,2929,1747,1705,1640,1446,1416,1381,1355,995,912$. ${ }^{1} \mathrm{H}$ NMR (300 MHz, $\left.\mathrm{CDCl}_{3}+\mathrm{CCl}_{4}\right): \delta$ 6.02-5.90 $(1 \mathrm{H}, \mathrm{m}), 5.85-5.69(1 \mathrm{H}, \mathrm{m}), 5.10-4.90(5 \mathrm{H}, \mathrm{m})$, $4.81(1 \mathrm{H}, \mathrm{s}), 2.76(1 \mathrm{H}, \mathrm{dd}, \mathrm{J}=10.5$ and $9.3 \mathrm{~Hz}), 2.65-2.15(4 \mathrm{H}, \mathrm{m}), 2.20\left(3 \mathrm{H}, \mathrm{s}, \mathrm{COCH}_{3}\right)$, 1.90-1.60 (4 H, m), $1.80\left(3 \mathrm{H}\right.$, s, olefinic $\left.\mathrm{CH}_{3}\right), 0.88\left(3 \mathrm{H}, \mathrm{s}\right.$, tert- $\left.\mathrm{CH}_{3}\right)$.

${ }^{13} \mathrm{C} \mathrm{NMR}\left(75 \mathrm{MHz}, \mathrm{CDCl}_{3}+\mathrm{CCl}_{4}\right): \delta 216.3(\mathrm{C}, \mathrm{C}=\mathrm{O}), 207.6(\mathrm{C}, \mathrm{C}=\mathrm{O}), 142.7(\mathrm{C}), 138.4(\mathrm{CH})$, $134.5(\mathrm{CH}), 118.4\left(\mathrm{CH}_{2}\right), 116.2\left(\mathrm{CH}_{2}\right), 115.0\left(\mathrm{CH}_{2}\right), 73.4(\mathrm{C}), 50.3(\mathrm{C}), 49.3(\mathrm{CH}), 42.7\left(\mathrm{CH}_{2}\right)$, $41.3\left(\mathrm{CH}_{2}\right), 31.5\left(\mathrm{CH}_{3}, \mathrm{COCH}_{3}\right), 31.2\left(\mathrm{CH}_{2}\right), 29.2\left(\mathrm{CH}_{2}\right), 24.1\left(\mathrm{CH}_{3}\right), 17.5\left(\mathrm{CH}_{3}\right)$.

HRMS: For $\mathrm{C}_{18} \mathrm{H}_{26} \mathrm{O}_{2} \mathrm{Na}(\mathrm{M}+\mathrm{Na})$, Calcd.: 297.1830. Found: 297.1840.

(+)-(1S,7S,10S)-7-Acetyl-1-methyl-10-isopropenylbicyclo[5.3.0]dec-3-en-8-one (25): $[\alpha]_{D}^{21} 349.0\left(c 1.0, \mathrm{CHCl}_{3}\right)$.

IR (neat): $v_{\max } / \mathrm{cm}^{-1} 3078,2960,2925,2850,1743,1702,1638,1453,1377,1356,1193,1143$, $1118,1083,895$.

${ }^{1} \mathrm{H}$ NMR $\left(300 \mathrm{MHz}, \mathrm{CDCl}_{3}+\mathrm{CCl}_{4}\right): \delta 5.49-5.40(2 \mathrm{H}, \mathrm{m}, \mathrm{H}-3$ and $\mathrm{H}-4), 5.05(1 \mathrm{H}, \mathrm{s}), 4.80(1 \mathrm{H}$, s), $3.36(1 \mathrm{H}, \mathrm{d}, \mathrm{J}=16.8 \mathrm{~Hz}), 3.30(1 \mathrm{H}, \mathrm{t}, \mathrm{J}=10.5 \mathrm{~Hz}), 2.51-2.08(3 \mathrm{H}, \mathrm{m}), 2.43(1 \mathrm{H}, \mathrm{d}, \mathrm{J}=7.8$ Hz), $2.39(1 \mathrm{H}, \mathrm{d}, \mathrm{J}=6.0 \mathrm{~Hz}), 2.11\left(3 \mathrm{H}, \mathrm{s}, \mathrm{COCH}_{3}\right), 1.97-1.75(2 \mathrm{H}, \mathrm{m}), 1.81$ (3 H, s, olefinic $\left.\mathrm{CH}_{3}\right), 0.81\left(3 \mathrm{H}, \mathrm{s}\right.$, tert $\left.\mathrm{CH}_{3}\right)$.

${ }^{13} \mathrm{C}$ NMR $\left(75 \mathrm{MHz}, \mathrm{CDCl}_{3}+\mathrm{CCl}_{4}\right): \delta 212.7(\mathrm{C}, \mathrm{C}=\mathrm{O}, \mathrm{C}-8), 204.2\left(\mathrm{C}, \mathrm{CH}_{3} \mathrm{C}=O\right), 142.1(\mathrm{C}$, $\left.C=\mathrm{CH}_{2}\right), 128.4(\mathrm{CH}), 127.1(\mathrm{CH}), 114.9\left(\mathrm{CH}_{2}, \mathrm{C}=\mathrm{CH}_{2}\right), 78.1(\mathrm{C}), 48.2(\mathrm{C}), 47.2(\mathrm{CH}), 40.3$ $\left(\mathrm{CH}_{2}\right), 35.8\left(\mathrm{CH}_{2}\right), 28.3\left(\mathrm{CH}_{3}, \mathrm{COCH}_{3}\right), 26.3\left(\mathrm{CH}_{2}\right), 24.9\left(\mathrm{CH}_{2}\right), 24.7\left(\mathrm{CH}_{3}\right), 19.2\left(\mathrm{CH}_{3}\right)$. HRMS: For $\mathrm{C}_{16} \mathrm{H}_{22} \mathrm{O}_{2} \mathrm{Na}(\mathrm{M}+\mathrm{Na})$, Calcd.: 269.1518 Found: 269.1517 .

S-13 to S-29: ${ }^{13} \mathrm{C}$ NMR spectra of the compounds $\mathbf{5 , 6 , 9 - 1 5 , ~ 1 8 - 2 5 . ~}$ 